\title{
La communication médiatique au service de l'environnement? la crise des déchets au liban
}

Media communication in the service of the environment? the waste crisis in Lebanon

Abi Karam Dana

\section{CpenEdition}

\section{Journals}

Édition électronique

URL : http://journals.openedition.org/ctd/1004

DOI : $10.4000 /$ ctd. 1004

ISSN : 2491-1437

Éditeur

Chaire Unesco Pratiques émergentes en technologies et communication pour le développement

Édition imprimée

Date de publication : 30 octobre 2016

ISBN : 2491-1437

Référence électronique

Abi Karam Dana, « La communication médiatique au service de l'environnement ? la crise des déchets au liban », Communication, technologies et développement [En ligne], 3 | 2016, mis en ligne le 30 octobre 2016, consulté le 10 décembre 2020. URL : http://journals.openedition.org/ctd/1004 ; DOI : https:// doi.org/10.4000/ctd. 1004

Ce document a été généré automatiquement le 10 décembre 2020.

Communication, technologies et développement 


\section{La communication médiatique au service de l'environnement? la crise des déchets au liban}

Media communication in the service of the environment? the waste crisis in Lebanon

Abi Karam Dana

\section{NOTE DE L'ÉDITEUR}

الإعلام في خدمة البيئة ؟ أزمة النفايات في لبنان

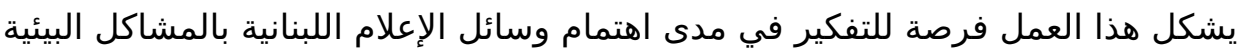

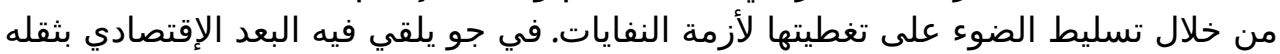

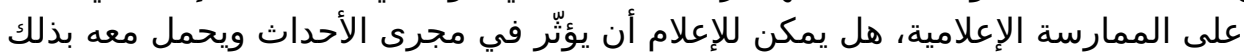

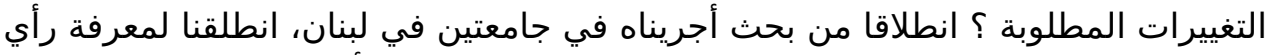

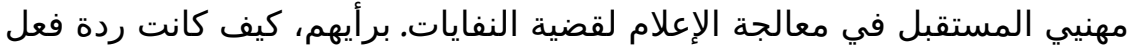

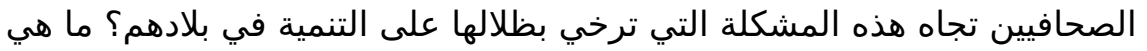

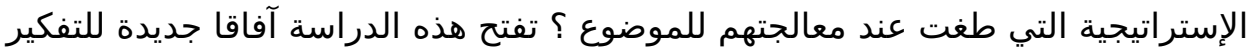

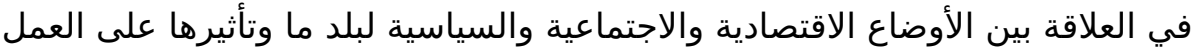
الإعلامي.

من ناحية أخرى، لقد وفّر تطور الوسائل التكنولوجية "مساحة عامة جديدة " سهّلت على اللى المامي

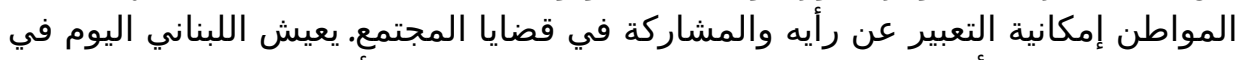

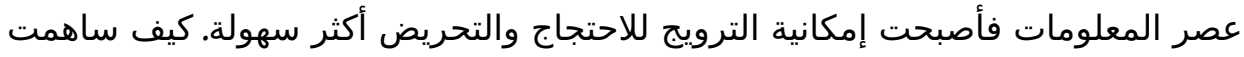

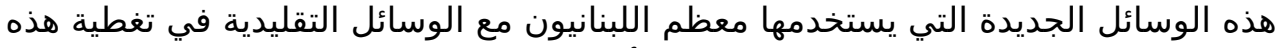

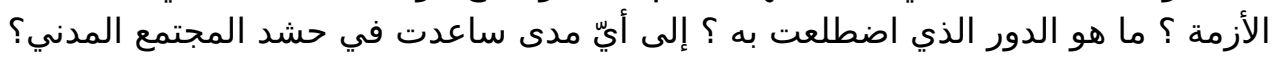
أزمة النفايات، البيئة، عقد إعلامي، الحدث، مدى استراتيجية الإعلام وجذب المجنب الإنتباه

1 Le développement vertigineux des réseaux de transmission électroniques envahit toutes les sphères de notre vie en société. Jamais la communication sociale n'a disposé, 
à travers l'histoire, d'aussi nombreux canaux que ceux qui sont les siens aujourd'hui. À côté des médias traditionnels, une nouvelle « sphère publique » voit le jour et permet la discussion et le traitement des questions environnementales. Les sites d'informations en ligne, les forums, les centres de recherches s'accompagnent d'une augmentation des blogs des journalistes et des lecteurs en ligne. Et pourtant, nos sociétés connaissent toujours des conflits, les problèmes politiques et écologiques s'accentuent, sans oublier la question d'hypermédiatisation et de conformisme. Ce qui amène D. Wolton à parler de contradictions culturelles, ou pour le dire autrement, d'un décalage entre les promesses et les réalités.

2 L'augmentation du nombre des supports assure-t-elle automatiquement un effet de diversification et une meilleure qualité de l'information, dans notre cas, environnementale? Les fonctions réellement assumées par les médias reflètent-elles les fonctions officiellement proclamées? En effet, la transformation des institutions médiatiques en industries ou l'œuvre en simple produit fait peser de nouvelles contraintes économiques sur les journalistes. Ils deviennent les maillons faibles de la chaîne de production. A l'encontre de la violence, du sexe, du sensationnel, l'environnement ne constitue pas une recette apte à séduire le public. En revanche, dans une société politisée comme au Liban, pourquoi le problème des déchets survenu en juillet 2015 est-il devenu l'objet de toutes les attentions? Commentles technologies de l'information et de la communication ont-elles participé à côté des médias traditionnels à la couverture de cette crise ? quels rôles ont-elles joué à ce niveau? Qu'est-ce qui caractérise, selon nos futurs journalistes, le traitement médiatique de cet événement particulier?

3 Les tentatives de réponse à l'ensemble de nos interrogations s'appuient sur une enquête quantitative menée auprès d'un échantillon de 35 étudiants en journalisme dont l'âge est compris entre 18 et 22 ans. Sur les sujets enquêtés, 33 sont de sexe féminin tandis que deux sont de sexe masculin. Ce qui s'explique par le nombre restreint de jeunes hommes inscrits dans ce domaine. Nous profitons de notre statut d'enseignante dans deux universités, l'Université libanaise de Beyrouth et l'Université Saint-Esprit de Kaslik, pour comprendre la manière dont nos étudiants jugent le traitement par les journalistes de la question des ordures, et leur façon à eux d'envisager leur propre conception de ce traitement. Auraient-ils fait la même chose s'ils en avaient eu le loisir ? Comment envisagent-ils d'exercer leurs talents vis-à-vis de ce problème qui touche au développement de leur pays? Les journalistes libanais contribuent-ils au développement de la société ou au renforcement de l'opinion existante ? Les réseaux sociaux ont-ils participé de manière active dans les processus de mobilisation de la société civile?

4 Notre travail se déploie en trois parties. La première nous donne l'occasion de faire un retour en arrière qui nous permettra de comprendre le contexte d'apparition de cette crise au Liban. Ensuite, nous profitons de la deuxième partie pour analyser la place occupée par la nouvelle technologie dans la vie des jeunes libanais en considérant cette dernière comme facteur de mobilisation sociale. Enfin, nous interrogeons la représentation médiatique de cette crise. Ce travail ne se situe pas à un niveau exclusivement empirique. Plusieurs concepts sont mobilisés, entre autres le contrat de communication, la crise, l'événement. Ils permettent une interprétation pertinente des données recueillies et assurent la compréhension de la pratique contemporaine de la production de l'information. 


\section{Les déchets ménagers, une crise de représentation}

Une fois de plus, la gestion politique libanaise et la conscience civique mettent en danger l'équilibre écologique du pays et la santé des citoyens. Nous partons de l'idée selon laquelle la crise des déchets que nous vivons n'est rien d'autre que notre propre produit. L'amoncellement sans cesse croissant des détritus dans les rues est une manifestation visible du lien existant entre les échecs répétés dans le dossier des déchets ménagers et la corruption qui sévit dans le pays. C'est donc dans notre rapport à la nature qu'il faut comprendre l'état actuel de la situation. Pour toutes ces raisons, nous pouvons affirmer que la crise dont nous parlons a un caractère doublement politique et sociétal. Dans ce cadre de pensée, l'environnement demande à être compris comme étant le produit de nos pratiques et nos représentations. Dans une interview réalisée le 21 janvier 2016 sur la chaîne LBC dans le programme KalemNes ou parole des gens, le ministre de l'Environnement Akram Chehayyeb déplore le fait que «même les ordures ont été confessionnalisées». S'y ajoutent, selon lui, les intérêts politiques et économiques des uns et des autres qui entravent la résolution du problème. La question qui se pose est de trouver des terrains qui servent de décharges temporaires aux ordures de la capitale et du Mont-Liban. Néanmoins, aucune localité n'est disposée à les accueillir.

Deux visions contradictoires ${ }^{1}$ inspirent les différentes approches qui permettent de situer la relation de l'homme à l'environnement. D'une part, une conception anthropocentrique de la Création, dans laquelle l'humanité est destinée à manipuler et à asservir tous les objets de la nature. D'autre part, une conception non anthropocentrique ou biocentrique qui invite l'homme à respecter les régulations et les diversités d'une nature vivante. Nous soutenons l'idée selon laquelle les problèmes environnementaux au Liban, parmi lesquels figurent les déchets, reflètent une vision anthropocentrique et s'enfoncent dans la structure de notre société et de notre vision $\mathrm{du}$ monde. D'ailleurs, les résultats de notre enquête viennent confirmer cette hypothèse, car 28 sur 35 des interrogés sont convaincus que la logique de domination perturbe la relation du Libanais à l'environnement. La crise des déchets au Liban questionne la conscience civique sur laquelle viennent se greffer la violation des lois et un appétit d'argent aux proportions extrêmes. Ainsi, parmi les 35 futurs journalistes, 32 considèrent que la mauvaise gestion politique n'est pas la seule à mettre en cause pour comprendre la situation. Il faut y ajouter l'indifférence du citoyen et son éducation. Le problème que nous vivons ne peut donc se réduire à des dimensions purement politiques. Ses racines plongent aussi dans le social. Nous suivons le raisonnement de Hicham Stéphane Afeissa selon lequel il y a crise

7 "d'abord et avant tout en ce sens où les interventions humaines dans la nature ont atteint un point critique $»^{2}$. Nous allons essayer dans ce qui suit de montrer en quoi cette véritable crise

8 traduit une vision anthropocentrique.

9 A la question de savoir pourquoi les Libanais en sont arrivés là, un bref retour en arrière s'impose. Dans un article publié dans L'Orient Le Jour le 1er août 2015, S. Baaklini nous propose un guide historique des principales étapes de la gestion calamiteuse des ordures dans le Liban de l'après-guerre. Au sortir de la guerre civile ${ }^{3}$, au début des années 1990, le problème des déchets ménagers fait figure de grand blessé. A Beyrouth, 
leur déversement dans la mer se développe à partir des années 1978: une grande décharge à l'ouest, dans le secteur de Normandie et un énorme dépotoir côtier à l'est, celui de Bourj Hammoud.

En $1997^{4}$, il existe trois centres de traitement pour les ordures du Grand Beyrouth : le dépotoir de Bourj Hammoud, l'incinérateur de la Quarantaine et celui d'Amrousieh. L'incendie volontaire de l'incinérateur d'Amrousieh au mois de mai 1997, par une population en colère, le met hors service pour plusieurs mois. Quant au dépotoir de Bourj Hammoud, il a été fermé le 20 juillet 1997 suite à un sit-in de la population. Le Liban connaît alors une véritable crise. Ce qui pousse le ministre de l'Environnement de l'époque, Akram Chehayeb, à adopter un "plan d'urgence ", toujours opérationnel aujourd'hui. Dans le cadre de ce plan, un nouveau contrat avec la compagnie Sukleen ${ }^{5}$ a été signé pour le ramassage, le nettoyage et le traitement des déchets de Beyrouth et du Mont-Liban, qui a fini par comprendre quelque 300 municipalités. C'est avec ce plan

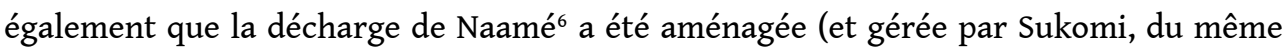
groupe que Sukleen) pour accueillir, selon le contrat initial, les déchets inertes (ni organiques ni recyclables) de cette région. Sa contenance ne devait pas excéder plus de deux millions de tonnes en dix ans; elle s'est retrouvée saturée en cinq ans. La décharge demeure sous-équipée en cheminées d'échappement des gaz, nécessaires à la purification du sol. Des promesses de recyclage, d'une meilleure gestion des déchets et de développement durable ont été faites à plusieurs reprises par les officiels, mais n'ont toujours pas été tenues. Exaspérés par les odeurs et la pollution dégagées par les montagnes de détritus, et craignant les retombées sur leur santé, les habitants des villages alentour estiment ce voisinage de plus en plus insupportable. Parallèlement à la saturation progressive de la décharge de Naamé, les plans nationaux de gestion des déchets, élaborés notamment par le ministère de l'Environnement avec le CDR, se sont succédé sans succès.

11 Le 17 juillet 2015, la fermeture de la décharge de Naamé et la fin du contrat de l'entreprise Sukleen qui gérait les poubelles contribuent à enfoncer le pays dans une crise toujours sans issue.

\section{«La révolution des ordures » à l'ère du numérique}

Les médias constituent un "espace public » central de discussion des problèmes environnementaux. Considérés comme un élément décisif pour la vie publique, culturelle et démocratique, ceux-ci permettent d'éveiller des débats en vue d'éclairer l'opinion. Nous considérons, dans l'exemple de la crise des déchets au Liban, les réseaux sociaux comme une nouvelle arène permettant à chaque citoyen d'y entrer et transformant, à travers la publication des opinions, la nation entière en juge. Les résultats de notre étude montrent que 29 des personnes interrogées sont connectées à internet à domicile et depuis le portable. Les autres se connectent depuis le portable seulement ou depuis les cybercafés. Ce nombre grandissant d'usagers du numérique permet d'affirmer que les jeunes Libanais se sont largement approprié cette technologie. De plus, tout le monde sans exception utilise les réseaux sociaux. D'un autre côté, 5 seulement des répondants affirment avoir suivi l'évolution de la crise des déchets uniquement sur les médias traditionnels contre 29 qui ont profité à la fois des réseaux sociaux et des médias traditionnels et un des réseaux sociaux simplement. Ce qui nous amène à affirmer que les réseaux sociaux sont devenus au Liban une source 
d'information très importante pour les jeunes. En fait, 13 futurs journalistes se basent seulement sur internet pour connaître l'actualité environnementale contre 12 qui ont recours à la télévision et à l'internet. Seule la partie restante considère les médias traditionnels (télévision/radio ou télévision/journal) suffisants pour avoir accès à l'information environnementale.

Dans le même ordre d'idées, nous avons essayé de savoir quel(s) usage(s) ces jeunes font du numérique. Parmi eux, 10 affirment que ce dernier constitue pour eux un outil pour suivre l'actualité ; 18 expliquent que le numérique leur permet de suivre l'actualité, mais aussi de se divertir et de communiquer avec les amis. Seuls 7 sur 35 ne se servent pas du numérique pour suivre l'actualité, mais pour communiquer avec les amis ou se divertir. Cette tendance de recherche des informations d'actualités sur le Web oblige les journaux à éditer en ligne. En effet, 26 interrogés déclarent lire la version électronique du journal. Nous partageons le constat de Dominique Augey qui explique que "l'apparition des TIC modifie profondément la production d'un journal qui utilise désormais des supports numériques ${ }^{7} »$.

Une enquête menée par Ipsos-Stat ${ }^{8}$ a montré que le taux de pénétration des utilisateurs des réseaux sociaux s'est élevé à $87 \%$ au Liban en 2013, contre $76 \%$ un an auparavant. Facebook fait fureur au Pays du Cèdre, avec un taux de pénétration de $98 \%$ en 2013, un pourcentage demeuré inchangé par rapport à 2012. La part des utilisateurs de Twitter par rapport à la base totale de ceux qui utilisent les réseaux sociaux était de $28 \%$ en 2013 au Liban, contre $18 \%$ en 2012. La part des détenteurs libanais de téléphone intelligent, ou smartphone, du total de la population a représenté $63 \%$ en 2013, alors qu'elle n'était que de $36 \%$ un an auparavant. Il est bon de souligner que $85 \%$ des détenteurs de smartphones au Liban utilisent le service 3G contre $74 \%$ en 2012. Cette enquête a été effectuée sur base d'un échantillon de 2500 Libanais.

La place grandissante occupée par les réseaux sociaux invite à se poser de nombreuses questions sur le rôle de ces nouveaux médias. Lors d'une conférence-débat organisée le 23 octobre 2015 à l'Institut des sciences politiques (ISP) de l'Université Saint-Joseph, Jacques Gerstlé se demande, à ce niveau, si internet revitalise la démocratie, ou s'il n'est qu'un prolongement des médias traditionnels et ne change par conséquent pas grandchose. Pour répondre à cette question, il dégage deux scénarios possibles dans le cas du Liban : soit les jeunes réussissent à capter le rejet actuel des hommes politiques et faire émerger de nouveaux leaders, soit les structures sociopolitiques traditionnelles conservent leur contrôle et bloquent tout changement par leur mainmise confessionnelle.

16 Concernant le cas particulier de la crise des déchets, 31 étudiants sur 35 pensent que les réseaux sociaux ont aidé à la promotion d'une culture de protestation pendant cette période. Dans un pays sans président depuis la fin du mandat de Michel Sleiman, le 25 mai 2014, et confronté à une situation économique difficile, la problématique des déchets est la goutte qui va faire déborder le vase.

Quelques jours après le début de la crise, les Libanais n'hésitent pas à exprimer leur mécontentement face à la situation sur les réseaux sociaux, notamment sur Twitter. Une vague d'indignation populaire se traduit par la publication de photos et des jeux de mots qui dessinent l'image de la question. Le Libanais Imad Mattar écrit sur un cliché rassemblant les députés et les éboueurs : "Quand vous réalisez que les éboueurs sont plus importants que nos députés ». Même ton ironique du côté du citoyen Nader Dagher qui présente les colonnes de Baalbeck, aux couleurs de sacs poubelles, désormais 
surnommés «Baalbag», un jeu de mots combinant "Baalbeck» et «bags " (sacs en anglais). La liste des pépites de cette crise vue par les twittos du pays est longue. Ralph Nader publie une photo officielle du gouvernement, en présence de l'ancien président de la République, Michel Sleiman, dans laquelle l'ordinateur demande à l'utilisateur s'il souhaite « jeter à la poubelle » l'image. De son côté, Fouad Khreis présente la chanteuse libanaise Najwa Karam, debout devant un amas de détritus, chantant à un éboueur : « Si tu savais ce qui s'est passé en ton absence... des déchets, des saletés, une situation lamentable ", en allusion au titre de sa chanson Si tu savais ce qui s'est passé en ton absence.

Les responsables politiques se servent, eux aussi, des réseaux sociaux pour commenter la situation. Le président Michel Sleiman écrit sur son compte twitter le 21 juillet 2015 : "La politique est dans la rue, les déchets sont dans la rue, et la rue rejette la paralysie».

La floraison de nouveaux médias permet donc l'éclosion d'un nouvel espace de discussion au cœur duquel de nouvelles identités peuvent se forger. Loin de nous l'idée de défendre la toute puissance des nouvelles technologies d'information et de communication dans la mobilisation de l'opinion publique, mais nous insistons sur leurs rôles d'instrument de communication et de critique des problèmes de société. Ceux-ci ouvrent la voie à une participation et une interaction pour les citoyens. Cela nous fait penser aux travaux réalisés sur la place occupée par les réseaux sociaux au cours des révolutions et leur importance dans le processus de changement politique et social. Les bouleversements et mouvements de contestation dans le monde arabe constituent ainsi un cadre de réflexion particulièrement intéressant. De la

« révolution du jasmin » en Tunisie au «printemps arabe » en Égypte et la chute du colonel Khadafi en Libye, les nouvelles technologies ont constitué un moteur de ces mouvements.

Pouvons-nous voir en ces technologies un forum qui nous permet d'entendre l'écho de toutes les voix publiques?

L'activisme en ligne s'accompagne d'une mobilisation et d'actions sur le terrain. Les Libanais descendent dans les rues pour exprimer leur ras-le-bol. La crise du ramassage des ordures ménagères donne naissance à la campagne baptisée Tolit rihetkoun, qui signifie «Vous puez». Des dizaines de manifestants accusent le gouvernement du premier ministre Tammam Salam d'être responsable de cette crise. Ils exigent sa démission ainsi que celle du ministre de l'Environnement Mohammad Machnouk, et réclament un système fondé sur le tri et le recyclage. Ce mouvement de protestation dénonce aussi les pénuries d'eau, les coupures d'électricité et le blocage des institutions. Une liste, non exhaustive, qui reflète le vrai visage d'un système politique gangrené par la corruption et le clientélisme. Ceci sans oublier le manque de responsabilité ou la tendance à se dérober à ses responsabilités.

\section{La médiatisation de la crise des déchets}

23 L'amoncellement des ordures sur les bords des rues ne constitue pas seulement une catastrophe écologique, mais pose un sérieux problème sanitaire. La lecture de quelques titres parus dans des quotidiens nationaux entre juillet et décembre 2015 montre que les journalistes consacrent plusieurs articles à ce sujet. Sous le titre «Les déchets, un enjeu de santé publique sur le long terme", Nada Merhi évoque, dans L'Orient Le Jour du 22 juillet, les dangers de leur accumulation sur la santé. D'après un 
spécialiste des maladies infectieuses, les déchets sont organiques dans leur majorité. Ils se décomposent et produisent un liquide qui s'infiltre dans le sol et facilite la prolifération des rongeurs, et par conséquent celle des germes d'infection intestinale, comme la salmonella.

En ce qui concerne l'incinération sauvage par certaines municipalités, le spécialiste explique que cette mesure permet de lutter contre le danger organique des déchets, mais elle crée un autre problème, celui de la pollution atmosphérique et des maladies qu'elle peut engendrer ou exacerber. L'attention portée à la dimension sanitaire du problème s'explique, selon nous, par un nombre de critères qui président aux choix qu'opère l'instance médiatique. Néanmoins, nous ne pouvons avancer dans la compréhension du fonctionnement du champ médiatique sans prendre appui sur une définition claire du concept d'événement. La question qui se pose consiste à savoir si l'événement est une donnée de la nature ou au contraire un objet de construction médiatique. "Est-ce parce qu'un événement est important qu'il focalise l'attention des médias ou est-ce parce qu'il est médiatisé qu'il devient retentissant? »".

Deux hypothèses contradictoires sont à la base de toute définition de ce concept : l'approche constructiviste et celle naturaliste. Dans une thèse soutenue en $2013^{10}$, nous avons essayé de montrer que ces deux visions se complètent. En effet, l'approche constructiviste ne peut naître du néant et puise son existence d'une certaine réalité. Sur la base de cette hypothèse nous pouvons avancer plusieurs constatations. D'abord le contenu d'un journal est fondé sur un réel déjà présent et représente le reflet des faits qui surgissent dans l'espace public. Vu que ces faits sont tellement nombreux, les médias ne peuvent pas les traiter en totalité. Il s'agit donc toujours d'un choix opéré par le journaliste dans l'ensemble du réel. Le journal, doté d'un pouvoir de sélection et de représentation, fait en quelque sorte exister l'événement, et cela sous un format qui lui est propre. Les faits renvoient à l'ensemble des phénomènes qui surviennent dans notre quotidien alors que les événements n'en sont que la partie émergente à la surface des médias. "L'événement ne fait qu'un avec sa réflexion : la réflexion est constitutive de son concept. La conséquence en est que tout événement est orienté ". ${ }^{11}$

Il convient donc de se demander quelles sont les lois qui gouvernent la production journalistique. À côté de la vitesse, de la proximité, de l'actualité et de l'importance de l'événement, l'intérêt humain fait partie, à son tour, des principes de sélection adoptés par les médias. Plus de la moitié des répondants auraient privilégié la dimension sanitaire pour représenter cette crise.

De plus, la transformation de l'institution médiatique en entreprise économique laisse, à son tour, ses traces sur la qualité de l'information proposée. Les enjeux politiques et financiers qui animent l'autre côté du miroir médiatique façonnent la pratique contemporaine de la production d'information. Comme l'explique Anders Hansen, " other pressures on the journalist, when deciding on what to cover, relate to the political and economic allegiances of individual newspapers $»^{12}$.

Ce qui se traduit par la mise en place de stratégies de captation et de manipulation de l'opinion, seules capables d'assurer la rentabilité et la pérennité des industries médiatiques. Dès lors, nous pouvons aisément affirmer que les médias ne sont pas neutres face à l'information transmise. Dans un monde soumis actuellement à la loi du spectacle, ces derniers ne font que transformer les «œuvres de l'esprit » en simple marchandise qui a un coût et qui doit être vendu. Concernant la télévision, à titre d'exemple, Karl Popper explique que «la violence, le sexe, le sensationnel, sont des moyens 
auxquels les producteurs de télévision recourent le plus facilement: c'est une recette sûre toujours apte à séduire le public » ${ }^{13}$.

Les pressions économiques pesant sur les journalistes ont un impact quant à la rigueur journalistique et ne reflètent malheureusement pas les piliers de la profession. Comme l'explique P. Charaudeau ${ }^{14}$, la finalité du contrat de communication médiatique se trouve en tension entre deux visées qui correspondent chacune à une logique particulière: une visée de faire savoir, ou visée d'information; une visée de faire ressentir, ou visée de captation. La question qui se pose est de savoir, dans un environnement économique transformé, se trouvant en position permanente de concurrence, quelle visée domine le traitement médiatique des évènements, dans notre cas la crise des déchets. Les résultats du questionnaire prouvent que 26 des 35 étudiants interrogés sont convaincus que la stratégie de captation est celle qui occupe la plus grande importance dans la médiatisation de cet événement. De plus, 28 d'entre eux estiment que la couverture médiatique de la crise est loin d'être idéale, mais elle est

« moyenne ». D'un autre côté, parler de " contrat de communication » nous invite à nous demander en quoi les attentes supposées du public peuvent influencer la nature des enjeux médiatisés. Toujours selon P. Charaudeau, le contrat nécessite une connaissance de l'identité des partenaires de l'échange. Les discours médiatiques sont construits en fonction de l'identité supposée des récepteurs. Ce qui justifie, selon nous, le fait que 28 des 35 enquêtés pensent que les médias ont privilégié dans leur couverture médiatique la dimension politique du problème. Car la politique occupe le devant de la scène au Liban. Malheureusement, les traces des crises sécuritaires, économiques et politiques que le pays a traversées laissent leurs empreintes sur les préoccupations des médias et du peuple. Pour étayer ce raisonnement, nous avons demandé aux étudiants dans quel domaine ils aimeraient travailler à l'avenir : 12 affirment que la politique est le seul sujet qui les attire; 5 sont intéressés par la politique et le social ; 15 se préoccupent du social. La partie restante (3 étudiants) vise le social et l'environnement. Ce qui prouve que cette thématique n'est pas prioritaire chez le Libanais. L'environnement n'est pas pris en compte à rang égal avec la prestigieuse politique.

31 En conclusion, nous pouvons constater que les nouvelles technologies ne sontpas une baguette magique capable de proposer des solutions à tous les maux de la société. La problématique environnementale ne peut être pensée en dehors de l'action de l'homme qui occupe l'espace, le transforme et le gère. Cependant, dotées d'un pouvoir d'atteindre le plus grand nombre de récepteurs, ces technologies sont à la fois un moteur et un levier de mobilisation sociale.

Depluslamédiatisationdelacrisedesdéchetsnousapermisd'interrogerlesdéfisquimenacent la pratique journalistique. En effet, le choix opéré par l'instance médiatique est touché par l'environnement économique, politique et social qui l'entoure. L'analyse du processus de sélection de l'information et de sa représentation se fait en fonction des attentes présupposées des audiences concernées d'une part et des contraintes de production d'autre part. 


\section{BIBLIOGRAPHIE}

Abi Karam, Dana, Crise écologique et représentation médiatique le cas libanais dans sa presse écrite nationale, thèse de l'Université de Bordeaux 3, 2013.

Arquembourg-Moreau, Jocelyne, Le temps des événements médiatiques, éd. De Boeck, Bruxelles, 2003.

Afeissa, Hicham-Stéphane, Qu'est-ce que l'écologie ?, éd. Librairie philosophique J. Vrin, Paris, 2009.

Augey, Dominique, « Les journalistes : petits maillons au bout de la chaîne industrielle », Hermès 35, 2003.

Baaklini, Suzanne, En matière de communication politique les réseaux sociaux talonnent la télé, L'Orient Le Jour, 23 octobre 2015.

Charaudeau, Patrick, Les médias et l'information l'impossible transparence du discours, éd. De Boeck, Bruxelles, 2005.

Cox, Robert, Environmental communication and the public sphere, éd. Sage, California, 2010.

Figuié, Gérard, Le point sur le Liban 2008, éd. Maisonneuve et Larose, Beyrouth, 2008.

Hansen, Anders, Environment, media and communication, éd. Routledge, New York, 2010.

Mouillaud, Maurice et Tétu, Jean François, Le journal quotidien, éd. Presses Universitaires de Lyon, 1989.

Mokbel, Liliane, Réseaux sociaux. Les internautes libanais branchés. L’Hebdo Magazine, $\mathrm{n}^{\circ} 2944$, 11 avril 2014.

Popper, Karl in Zarka, Yves Charles, Démocratie et pouvoir médiatique, Cités 2/2002 ( $\left.\mathrm{n}^{\circ} 10\right)$, p. 119-129.

Wolton, Dominique, Penser la communication, éd. Flammarion, France, 1997.

\section{NOTES}

1. Abi Karam, Dana, Crise écologique et représentation médiatique le cas libanais dans sa presse écrite nationale, thèse de l'Université de Bordeaux 3, 2013, p. 102.

2. Afeissa, Hicham-Stéphane, Qu'est-ce que l'écologie?, éd. Librairie philosophique J. Vrin, Paris, 2009, p. 48.

3. Dont le gouvernement à travers le Conseil du développement et de la reconstruction CDR lui a confié la collecte des déchets et le nettoyage des rues depuis 1994.

4. La guerre civile éclate le 13 avril 1975 et entraîne le pays dans une tourmente de 15 années cauchemardesques.

5. Figuié, Gérard, Le point sur le Liban 2008, éd. Maisonneuve et Larose, Beyrouth, 2008, p. 456.

6. village au sud de Beyrouth.

7. Augey, Dominique, Les journalistes : petits maillons au bout de la chaîne industrielle, Hermès 35, 2003. 
8. Mokbel, Liliane, Réseaux sociaux. Les internautes libanais branchés. L'Hebdo Magazine, n²944, 11 avril 2014.

9. Arquembourg-Moreau, Jocelyne, Le temps des événements médiatiques, éd. De Boeck, Bruxelles, 2003, p. 7

10. Abi Karam, Dana, Crise écologique et représentation médiatique : le cas libanais dans sa presse écrite nationale, Op. cit., p. 22.

11. Mouillaud, Maurice et Tétu, Jean François, Le journal quotidien, éd. Presses

Universitaires de Lyon, 1989, p. 21.

12. Hansen, Anders, Environment, media and communication, éd. Routledge, New York, 2010, p. 94.

13. Popper, Karl in Zarka, Yves Charles, Démocratie et pouvoir médiatique, Cités $2 / 2002$ (n $\left.{ }^{\circ} 10\right)$, p. 119-129.

14. Charaudeau, Patrick, Les médias et l'information l'impossible transparence du discours, éd. De Boeck, Bruxelles, 2005, p. 70.

\section{RÉSUMÉS}

Ce travail constitue une opportunité pour réfléchir sur le rôle des médias libanais dans la prise en charge des problèmes environnementaux en interrogeant leurs perceptions et représentations de la crise des déchets. Dans un contexte où la dimension économique pèse très fortement sur la pratique médiatique, l'information diffusée est-elle susceptible d'influencer le cours des choses et d'induire, en conséquence, les changements requis? A partir d'une enquête dans deux universités au Liban, nous désirions connaître l'avis de futurs professionnels sur le traitement médiatique de la question des ordures. Comment, selon eux, les journalistes en poste ont-ils réagi face à ce problème qui touche au développement du pays? Quelle stratégie ont-ils privilégiée dans leur traitement? Une telle étude ouvre des voies originales pour penser les relations entre conditions économiques, sociales, politiques d'un pays et production médiatique.

D'un autre côté, l'essor des nouvelles technologies permet l'avènement d'un nouvel «espace public ». La prise de parole citoyenne est facilitée et la participation à la vie publique est assurée. Le Libanais évolue dans un environnement de surcharge informationnelle où la promotion d'une culture de la contestation devient de plus en plus facile. Comment ces moyens technologiques, utilisés par la majorité des Libanais, ont-ils participé à côté des médias traditionnels, à la couverture de cette crise ? Quels rôles ont-ils joué ? Jusqu'à quel point ont-ils contribué à la mobilisation de la société civile?

This work is an opportunity to reflect on the role of the Lebanese media in the management of environmental problems. It proceeds with an examination of their perceptions and representations of the waste crisis. At a time when the economic dimension plays a major role in media practice, could the information broadcasted cause the events to change course, thus bringing the much-needed changes? Through a survey conducted in two universities in Lebanon, we sought to take stock of the opinion of future professionals on how the media is tackling the garbage issue. In their opinion, how have the current journalists reacted to this problem, one that thwarts the country's development? For which strategy did they opt to address this matter? Such a study sheds light on new ways to reflect on the relationship between the 
country's economic, social and political conditions on the one hand and its media production on the other.

Furthermore, new emerging technologies provide a new "public space." This allows citizens to voice their opinion as it guarantees their participation in public life. Lebanese citizens are surrounded by information overloads; and in such an environment, the promotion of a culture of protest becomes easier. How have these technology gadgets used by the majority of the Lebanese people contributed alongside the traditional media to the coverage of this crisis? What role have they played? To what extent have they contributed to the mobilization of the civil society?

INDEX

Mots-clés : crise des déchets, environnement, contrat de communication, événement, stratégie d'information et de captation

Keywords : wastecrisis, environment, communication contract, event, information and attraction study

\section{AUTEUR}

\section{ABI KARAM DANA}

Université Libanaise et Université Saint-Esprit de Kaslik 